\title{
COVID-19 Infection and Acute Kidney Injury: Cause or Complication?
}

\author{
Matthias Arnaldo Cassia ${ }^{a, b}$ Roberta Casazza ${ }^{a, b}$ Pietro Napodano ${ }^{a}$ \\ Mario Cozzolino ${ }^{a, b}$

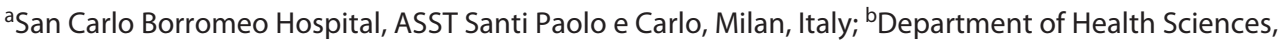 \\ University of Milan, Milan, Italy
}

\section{Keywords}

COVID-19 · Hyponatremia $\cdot$ Acute kidney injury $\cdot$ Renal replacement therapy · Acute interstitial nephritis

\begin{abstract}
Management of COVID-19 infection is the trend topic in the scientific community and case identification is a key step to contain the pandemic. While pneumonia and acute respiratory distress syndrome represent the typical severe manifestations of the disease, atypical presentations pose significant diagnostic and therapeutic challenges for physicians, especially when diagnostic tests are repeatedly negative. Clinical picture of COVID-19 patients is often complicated by bacterial infections or thrombotic events. Here, we present and discuss a case report identified in our center as example of a challenging diagnosis and 2 uncommon complications: severe hyponatremia and acute kidney injury requiring renal replacement therapy, caused by parenchymal damage and with a possible direct involvement of the virus.
\end{abstract}

C 2021 S. Karger AG, Basel

\section{Introduction}

Management of COVID-19 infection is the trend topic in the scientific community, and case identification is a key step to contain the pandemic. While pneumonia and acute respiratory distress syndrome represent the typical severe manifestations of the disease, atypical presentations pose diagnostic and therapeutic challenges for physicians. Here, we present a case report as example of uncommon complication: acute kidney injury (AKI) requiring renal replacement therapy (RRT).

\section{Case Report/Case Presentation}

An 83-year-old white woman was admitted due to cognitive impairment and cranial trauma resulting from a fall occurred 11 days before. The patient was also referred to be feverish and lamenting dysuria in the few days after the fall; of note, her husband had died with COVID-193 weeks before. Her history was positive only for hypertension. She was being treated at home with aspirin, ACE inhibitor, calcium antagonist, and thiazide diuretics. The patient was lethargic, whereas a review of systems performed negative, except for slight bilateral pretibial edema. Her blood pressure was $130 / 80 \mathrm{~mm} \mathrm{Hg}$, temperature was $36.4^{\circ}$, heart rate was 100 beats per minute, and oxygen saturation was $88 \%$ breathing in am-

\footnotetext{
Karger"
} 
Table 1. Laboratory tests over the follow-up of our patients at different time points

\begin{tabular}{lccrr}
\hline Variable & $\begin{array}{l}\text { Reference } \\
\text { range }\end{array}$ & $\begin{array}{l}\text { On } \\
\text { admission }\end{array}$ & $\begin{array}{l}\text { Before start } \\
\text { of RRT }\end{array}$ & $\begin{array}{c}\text { Last } \\
\text { follow-up }\end{array}$ \\
\hline Hemoglobin, g/dL & $10.9-15.5$ & 11.2 & 7.7 & 7.4 \\
White cell count, per $\mu \mathrm{L}$ & $4,400-11,300$ & 32,790 & 26,540 & 7,340 \\
Platelet count, per $\mu \mathrm{L}$ & $150,000-350,000$ & 141,000 & 174,000 & 124,000 \\
C-reactive protein, mg/dL & $0.03-0.50$ & 18.05 & 18.78 & 4.3 \\
Procalcitonin, $\mu$ g/L & $<0.50$ & 31.1 & 11.9 & 0.84 \\
Creatinine, mg/dL & $0.50-0.90$ & 5.7 & 6.03 & 4.2 \\
Serum urea, mg/dL & $21-43$ & 255 & 376 & 173 \\
Sodium, mmol/L & $136-145$ & 109 & 142 & 135 \\
Potassium, mmol/L & $3.4-4.5$ & 5.93 & 5.2 & 3.3 \\
Glucose, $\mathrm{mg} / \mathrm{dL}$ & $65-100$ & 348 & 319 & 253 \\
LDH, U/L & $120-250$ & 308 & 13,880 & 11,000 \\
D-dimer, $\mathrm{ng} / \mathrm{mL}$ & $<270$ & 16,329 & & 255 \\
\hline
\end{tabular}

RRT, renal replacement therapy.

bient air. Diuresis was preserved and monitored through a catheter collecting pyuria.

Blood tests showed increased inflammatory markers together with AKI and severe hyponatremia; D-dimer was highly increased. ABG showed mild hypoxia and signs of peripheral hypoperfusion; no respiratory insufficiency was observed (P/F 300). A SARSCoV2 nasopharyngeal swab and blood cultures tested negative, whereas urine culture tested positive for E. coli. Chest X-ray showed bilateral ill-defined consolidations at the pulmonary basis, with an associated left pleural effusion. Chest CT confirmed x-ray results: of note, no ground-glass areas were found. Abdomen and brain CT were normal. Ultrasonography did not show urinary tract dilation and no signs of fluid overload.

The first diagnosis hypothesized was a urinary tract infection (UTI) complicating the fall. In the elderly population, falls are a renowned source of morbidity and mortality and are followed up in up to $10 \%$ of cases by serious sequelae [1]. Particularly, a fall causes prolonged bed rest, decreased hydration, and may induce progressive cognitive impairment, if brain trauma is associated.

Our patient had signs of severe sepsis: leukocytosis, tachycardia, and severe AKI. In complicated UTI, up to $12.3 \%$ of patients develop AKI, with the elderly more susceptible to kidney damage [2]. Moreover, cognitive impairment is extremely common, and sepsis-associated encephalopathy targets up to $50 \%$ of patients with severe sepsis [3]. However, hyponatremia contributes to the neurological symptoms of our patient, and sepsis-induced SIADH can be a complication in up to $20 \%$ of patients admitted in an ICU [4].

However, pulmonary symptoms and radiologic evidence of consolidation could be a sign of pulmonary infection, evolved as severe sepsis; moreover, SIADH is a more common complication of pneumonia, rather than UTIs, independently from the severity. As such, a bacterial or a viral pneumonia could be not ruled out [5].

Considering all patients as potentially infected is crucial, especially in case of contact with a confirmed case. Typical symptoms include fever, dry cough, and fatigue; some patients may develop pneumonia, and $10-20 \%$ of severe cases evolve into acute respiratory distress syndrome. White cell count is usually normal, and there may be lymphopenia. Inflammatory markers are usually elevated, and a high procalcitonin level may indicate a bacterial coinfection. ALT/AST, D-dimer, CPK, and LDH levels may be elevated [6].

Laboratory tests and the history of contact with a confirmed case make the diagnosis of COVID-19 possible. High WBC and PCT, despite being atypical, can be explained by a coexisting UTI. Desaturation, mild hypoxia, and radiological features are consistent with COVID-19 pneumonia. Moreover, a negative nasopharyngeal swab does not allow ruling out the diagnostic hypothesis, due to low sensitivity of this test, especially in patients with mild respiratory symptoms (70\% or lower) $[7,8]$.

Physicians face challenging diagnosis also with atypical presentations of COVID: in elderly or immunocompromised patients' fever may be absent, and extrapulmonary manifestations are documented. Up to a third of hospitalized patients develop neurological symptoms such as confusion and headache or more specific manifestations, like seizure or cerebrovascular events [9]. Cognitive impairment in our case has been attributed to SIADH-related hyponatremia, as previously stated. Even if there are no data linking directly SIADH to COVID pneumonia, viral pneumonias are a frequent cause of hyponatremia.

Some data report kidney involvement as well among manifestations of COVID-19, as AKI or as isolated urinary anomalies. In observational data from Wuhan, AKI affected 25-29 percent of patients who were critically ill [10] and renal complications were associated with higher mortality. Most of the time, AKI is reasonably due to hemodynamic changes after systemic cytokine release syndrome, but direct cytotoxicity of the virus could be involved as well [11].

Kidney histopathology was examined in an autopsy series of 26 patients who died with COVID-19: all patients had evidence of acute tubular injury (of varying severity); erythrocyte clusters and pigmented casts were also present. SARS-CoV2 uses ACE2 receptors to enter target cells, and autoptic studies showed viral replica- 
tion inside tubular cells [12]. Among tubular kidney diseases, a number of viruses can cause an acute interstitial nephritis, which clinically manifests itself as an AKI with preserved diuresis [13]. Pyuria can be observed in up to $50-60 \%$ of cases, and a urine test does not show remarkable anomalies, except urinary WBC.

The patient was started on i.v. ceftriaxone and sodium supplement, with correction of hyponatremia and decrease of inflammatory markers; despite that, renal function did not respond to treatment. A second nasopharyngeal swab for SARS-CoV2 tested negative. After 5 days of antibiotics treatment, procalcitonin kept decreasing, whereas C-reactive protein plateaued; moreover, even with valid diuresis and diuretic stimulus, renal function kept worsening; therefore, the patient was started on RRT. The third nasopharyngeal swab performed for SARS-CoV2 resulted positive.

After this, the patient was isolated and needed RRT (hemodialysis) for 2 sessions, after which renal function partially recovered (eGFR $18 \mathrm{~mL} / \mathrm{min}$ ). At the end of our follow-up, the patient had a valid biochemical response to treatment, but the general conditions remained critical (Table 1 ).

\section{Discussion/Conclusion}

This article covers 2 major topics: first one is the necessity of COVID-19 diagnosis. In our case, final diagnosis was delayed due to a false-negative test of the first and second nasopharyngeal swabs and the overlap in presenting symptoms; we would like to stress the importance of a clinically driven approach and diagnosis, eventually supported by laboratory finding. Bacterial coinfection in this case is a confounding factor, but one must note that the rate of coinfection in this disease is particularly high (up to $10-20 \%$ as described in a Wuhan report) [14]. Differential diagnosis was in the end driven by the profile of biochemical alterations and especially the different response of inflammatory markers to the antibiotic treatment.

The second topic worth noting is the renal involvement in SARS-CoV2: most renal function alterations could be attributable to hemodynamic changes during the massive systemic inflammatory response, typical of COVID-19. In most severe cases, especially if a severe sepsis superimposes, AKI evolves to acute tubular necrosis [15]. It differentiates from our case as in most cases, acute tubular necrosis starts as an oligo-anuric AKI, whereas our case maintained diuresis from the very beginning. However, being this a possibility, the other one is that COVID-19 might have direct involvement in inducing kidney inflammation as acute interstitial nephritis. There is no solid evidence in the scientific literature, but the indifferent urine test and preserved diuresis, which are markers of AIN, are suggestive. Moreover, there was no rapid recovery of renal function with re- sponse to infection; on the contrary, after a few days of dialysis, renal function stabilized. No histologic assessment was performed due to the critical conditions and the prophylactic anticoagulant treatment. Keeping with this, no treatment was started due to bacterial coinfections and the patient's comorbidities.

In conclusion, we would like to stress out the importance of careful review of clinical symptoms before ruling out a COVID-19 infection and, second, to suggest that kidney could be directly involved among manifestations of COVID-19. More cases would help treatment and expand our knowledge, especially regarding the prognosis of renal involvement.

\section{Statement of Ethics}

This study complies with the ethical principles highlighted in the World Medical Association Declaration of Helsinki. Written informed consent has been obtained by the patient, provided grant of anonymity in the reporting of the case. Being a case report, the need for approval for this study was waived by our Institution's Ethical Committee (Ethical Committee of the ASST Santi Paolo e Carlo (Milan, Italy)

\section{Conflict of Interest Statement}

Prof. Mario Cozzolino is a member of the board of Blood Purification in the "Hemodialysis" section. The other authors have no conflicts of interest to declare.

\section{Funding Sources}

No funding was required for the draft of this study.

\section{Author Contributions}

Matthias Cassia and Roberta Casazza wrote the manuscript. Pietro Napodano and Mario Cozzolino supervised and reviewed the draft before submission.

\section{References}

1 Fuller GF. Falls in the elderly. Am Fam Physician. 2000 Apr 1;61(7):2159-68.

2 Hsiao CY, Yang HY, Hsiao MC, Hung PH, Wang MC. Risk factors for development of acute kidney injury in patients with urinary tract infection. PLoS One. 2015;10(7): e0133835.

3 Chung HY, Wickel J, Brunkhorst FM, Geis C. Sepsis-associated encephalopathy: from Delirium to Dementia? J Clin Med. 2020 Mar; 9(3):703. 
4 Ansley Buffington M, Abreo K. Hyponatremia: a review. J Intensive Care Med. 2016 May;31(4):223-36.

5 George L, Milionis HJ, Elisaf M. Hyponatremia in patients with infectious diseases. J Infect. 2011 Nov;63(5):327-35.

6 Ge H, Wang X, Yuan X, Xiao G, Wang C, Deng $\mathrm{T}$, et al. The epidemiology and clinical information about COVID-19. Eur J Clin Microbiol Infect Dis. 2020 Jun;39(6):1011-9.

7 Patel R, Babady E, Theel ES, Storch GA, Pinsky BA, George KS, et al. Report from the American society for microbiology COVID-19 international summit, 23 march 2020: value of diagnostic testing for SARS-CoV-2/ COVID-19. mBio. 2020 Mar-Apr; 11(2): e00722-820.
8 Zitek T. The appropriate use of testing for COVID-19. West J Emerg Med. 2020 Apr 13; 21(3):470-2.

9 Ahmad I, Rathore FA. Neurological manifestations and complications of COVID-19: a literature review. J Clin Neurosci. 2020 May;77: 8-12.31078X

10 Yang X, Yu Y, Xu J, Shu H, Xia J, Liu H, et al Clinical course and outcomes of critically ill patients with SARS-CoV-2 pneumonia in Wuhan, China: a single-centered, retrospective, observational study. Lancet Respir Med. 2020;8:475.

11 Ronco C, Reis T. Kidney involvement in COVID-19 and rationale for extracorporeal therapies. Nat Rev Nephrol. 2020 Jun;16(6):30810.
12 Alhenc-Gelas F, Drueke TB. Blockade of SARS-CoV-2 infection by recombinant soluble ACE2. Kidney Int. 2020 Jun;97(6):1091-3.

13 Bruggeman LA. Common mechanisms of viral injury to the kidney. Adv Chronic Kidney Dis. 2019 May;26(3):164-70.

14 Zhou F, Yu T, Du R, Fan G, Liu Y, Liu Z, et al. Clinical course and risk factors for mortality of adult inpatients with COVID-19 in Wuhan, China: a retrospective cohort study. Lancet. 2020 Mar 28;395(10229):1054-62.

15 Kundert F, Platen L, Iwakura T, Zhao Z, Marschner JA, Anders HJ. Immune mechanisms in the different phases of acute tubular necrosis. Kidney Res Clin Pract. 2018 Sep; 37(3):185-96. 